\title{
Commentary
}

\section{Lipidomics and Nutrylipidomics in Oncology: Review of the Literature}

\author{
Virgili $E^{1^{*}}$, Emili $\mathbf{R}^{2}$, Hammarberg Ferri I ${ }^{7}$, Palermo $F^{3}$, Calza L ${ }^{4}$, Gardarelli $M^{5}$, Spina $\mathbf{M}^{6}$ \\ ${ }^{1}$ Outpatient Nutritionist, Porto Sant'Elpidio, Italy \\ ${ }^{2}$ Department of Oncology, Urbino Hospital S.Maria, Italy \\ ${ }^{3}$ School of Biosciences and Veterinary Medicines, University of Camerino, Italy. \\ ${ }^{4}$ Outpatient Nutritionist, Fermo, Italy \\ ${ }^{5}$ Outpatient Medical Doctor, Integrative Medicine, Ancona, Italy \\ ${ }^{6}$ Laboratorio analisi Dr.Fioroni, San Benedetto del Tronto, Italy \\ ${ }^{7}$ Outpatient Oncologist, Bologna, Italy
}

*Corresponding Author: Dr. Edy Virgili. Outpatient Nutritionist, Porto Sant'Elpidio, Italy, E-mail: info@edyvirgili.it

Received: 23 November 2018; Accepted: 04 December 2018; Published: 17 December 2018

\section{Lipidomics and Nutrylipidomics in Oncology}

Like the other "Omics" Sciences (e.g. Genomics, Proteomics) dealing dynamically with molecules existing in living organisms, Lipidomics allows to evaluate, on different biological matrices, the fundamental units of the fatty acid classes composing our cell membranes: SFA (Saturated Fatty Acids), MUFA (Mono Unsaturated Fatty Acids), PUFA (Poly Unsaturated Fatty Acids). It studies not only the structure, but also the function and the variations (relationships between fatty acids) that are determined in different physio-pathological conditions, putting in close relation the membrane components with the nutritional status of the patient and with his/her metabolic dysfunctions (hormonal changes, insulin resistance, obesity etc.). In recent scientific studies, the crucial effect of fatty acids, linked to the correct functioning of ion channels and to the correct transfer of biologically active molecules through the membrane has been increasingly highlighted. It is evident how the organization of the membrane does not only produce a structural effect, but rather is the key point of the regulation and calibration of the whole cellular functioning. For this reason, the membrane can be considered as a metabolic pacemaker: this compartment has not anymore the role of a passive spectator, but even becomes an active protagonist in the life and destiny of cells. In recent years, the scientific literature has underlined how through the lipidomics on erythrocyte membrane (red blood cell), very sturdy indices can be used to identify food shortages, nutritional deficits, metabolic dysfunctions. Among these indices, the Omega-3 Index is considered one of the main biomarkers for cardiovascular pathologies and 
recently it has been related to the increase of oncological diseases. This value is given by the sum of the Omega 3 PUFAs (DHA + EPA, the Omega 3 from fish) and indicates if the nutritional intake is deficient, good or optimal, according to the International Guidelines. The dietary intake is essential and crucial because PUFAs, unlike SFA and MUFA, can not be synthesized endogenously, but they can only be introduced with the diet, hence the definition of Essential Fatty Acids (EFA). Another key index is the ratio between Omega-6/Omega-3 and pro-inflammatory Omega-6 (such as Arachidonic Acid), considered today the most significant test to detect the persistence of silent inflammation. While the ratio between saturated and unsaturated fats is progressively improving within the population, the ratio of PUFA Omega- 6 to Omega-3, whose ideal proportion is between 4:1 and 2:1, is currently unbalanced; in fact FDA highlights an average ratio of 25:1 in the ordinary nutrition (in Europe we are around 15:1). Identifying an optimal lipid pattern can be of great help in the management of many serious diseases [1-4].

Recently it has been observed how the variations in the relationships between MUFA and SFA (fatty acids that can undergo strong endogenous variations) are the indicators of metabolic imbalances such as obesity, insulin resistance and are the response to oncological signals for some types of tumors. Lipidomics is an emerging approach in the characterization of the tumor with regard to its classification and its differentiation with respect to healthy cells. Tumor cells use fatty acids to synthesize membranes, as an energy reserve and for the production of signaling molecules, with more complex metabolic mechanisms than those used for glucose [5-8].

In tumor cells there is an increased rate of lipid and cholesterol synthesis. Fatty acids are necessary for the proliferation of tumor cells and metabolic pathways that allow the growth of the tumor, therefore, they represent a new and selective target for oncological therapies. However, it is necessary to specifically understand the metabolism of lipids in tumor cells in different types of neoplasia. Unfortunately, lipid metabolism is complex, with many feedback mechanisms and metabolic steps. Moreover, most of the lipid metabolic enzymes have multiple isoforms active on various processes and may have different localization both at cellular and tissue level. Some studies confirm the alteration of lipid composition in the cell membranes of tumor cells; other studies focus on these alterations to understand diseases and to discover their potential as markers or therapeutic targets. For example, eicosanoids certainly play a role in the neoplastic transformation of cancer cells (proliferation, motility, migration, invasion, apoptosis, metastasis and angiogenesis). Some studies demonstrate that, through the blocking of the receptor for eicosanoids; through the overexpression of enzymes metabolizing eicosanoids and though the use of endogenous anti-inflammatory lipids, it is possible to inhibit the inflammation associated with the tumor and, thus, to have an anti-tumor effect [9-14].

Ways of communication between cells and some prostaglandins leading to the escape of cancer cells from the immune system are known. In cancer patients there are different lipid species in the cell lines of tissues, plasma, serum and urine and this can become an early diagnostic tool. This diagnostic power has been widely documented for phosphatidylcholines, phosphatidylethanolamine, phosphatidylinositol, lysophosphatidylcholine and sphingolipids. In lung and breast cancer studies, it appears that there are higher levels of glycerophospholipids and sphingolipids compared to healthy controls. Furthermore, high levels of glycerophospholipids appear to be related to 
negative prognostic factors suggesting a potential role in tumor progression. Ovarian cancer appears to be particularly related to elevated levels of lysophosphatidic acid and lysophosphatidylinositol; in particular, lysophosphatidic acid stimulates cell proliferation and migration. In pancreatic tumors, there is an altered production pathway of ceramides, which are different from those of healthy controls. The ceremids have an important role in tumor suppression as they amplify the signals leading to apoptosis, autophagy and cell cycle arrest. Nutrition plays a crucial role in this area, because fats used for the various metabolic pathways come from food; in particular, the contribution of omega-3 fatty acids is essential, but increasingly lacking in Western nutrition. There is not only scientific evidence on the therapeutic role of omega 3 against certain types of cancer, but also on improving the efficacy and tolerability of chemotherapy (e.g. suppression of NF-kB, activation of AMPK / SIRT1, modulation of cyclooxygenase activity, activation of anti-inflammatory lipid mediators such as resolvins and maresins). Lately we are also evaluating the multiple benefits of diomogammalinolenic acid, which exerts an anti-proliferative action through the reduction of free radicals, lipid peroxidation and the synthesis of inflammatory prostaglandins, influencing growth, apoptosis and cell differentiation. Lastly, there are studies showing that the diet is able to remodel the plasma membrane structure; as a consequence, an unbalanced diet can modify membrane functions, such as homeostasis and signaling functions, causing cellular aberrations [15-19].

\section{References}

1. Greene ER, Huang S, Serhan CN, et al. Regulation of Inflammation in Cancer by Eicosanoids. Prostaglandins Other Lipid Mediat 96 (2011): 27-36.

2. Zarate R, El Jaber-Vazdekis N, Tejera N, et al. Significance of long chain polyunsaturated fatty acids in human health. Clin Trans Med 6 (2017): 25.

3. Ebadi M, Field CJ, Lehner R, et al. Chemotherapy diminishes lipid storage capacity of adipose tissue in a preclinical model of colon cancer. Lipids in Health and Disease 16 (2017): 247.

4. Erazo-Oliveras A, Fuentes NR, Wright RC, et al. Functional link between plasma membrane spatiotemporal dynamics, cancer biology, and dietary membrane-altering agents. Cancer and Metastasis Reviews 37 (2018): 519-544.

5. Fuentes NR, Salinas ML, Kim E, et al. Emerging Role of Chemoprotective Agents in the Dynamic Shaping of Plasma Membrane Organization. BBA-Biomembranes 1859 (2016): 1668-1678.

6. Currie E, Schulze A, Zechner R, et al. Cellular Fatty Acid Metabolism and Cancer. Cell Metab 18 (2013): 153-161.

7. Huerta-Yepez S, Tirado-Rodriguez AB, Hankinson O. Role of diets rich in omega-3 and omega-6 in the development of cancer. Bol Med Hosp Infant Mex 73 (2016): 446-456.

8. Fuentes NR, Kim E, Fan YY, et al. Omega-3 fatty acids, membrane remodeling and cancer prevention. Molecular Aspects of Medicine 64 (2018): 79-91.

9. Rohrig F, Schulze A. The multifaceted roles of fatty acid synthesis in cancer. Nature Reviews Cancer 16 (2016): 732-749.

10. Wang X, Lin H, Gu Y. Multiple roles of dihomo-g-linolenic acid against proliferation diseases. Lipids in 
Health and Disease 11 (2012): 25.

11. Zhou P, Li B, Liu B, et al. Prognostic role of serum total cholesterol and high-density lipoprotein cholesterol in cancer survivors: A systematic review and meta-analysis. Clinica Chimica Acta 477 (2018): 94-104.

12. Francesca Perrotti, Consuelo Rosa, Ilaria Cicalini, et al. Advances in Lipidomics for Cancer Biomarkers Discovery. Int J Mol Sci 17 (2016): 1992.

13. Carla Ferreri, Consiglio Nazionale delle Ricerche. Membrana cellulare e Lipidomica. Bologna (2012).

14. William S Harris. Omega-3 Fatty Acids and Cardiovascular Disease: A Case for Omega-3 Index as a New Risk Factor. Pharmacol Res 55 (2007): 217-223.

15. Clemens von Schacky, William S Harris. Cardiovascular risk and the omega-3 index. Journal of Cardiovascular Medicine 1 (2007): 46-49.

16. Huerta-Yepez S, Tirado-Rodriguez AB, Hankinson O. Role of diets rich in omega-3 and omega-6 in the development of cancer. Bol Med Hosp Infant Mex 73 (2016): 446-456.

17. Carla Ferreri, Chryssostomos Chatgilialoglu. Role of fatty acid-based functional lipidomics in the development of molecular diagnostic tools. Expert Rev Mol Diagn 12 (2012): 767-780.

18. Puca AA, Andrew P, Novelli V, et al. Fatty acid profile of erythrocyte membranes as possible biomarker of longevity. Rejuvenation Research 11 (2008): 63-72.

19. Pala V, Krogh V, Muti P, et al. Erythrocyte Membrane Fatty Acids and Subsequent Breast Cancer: A Prospective Italian Study. Journal of the National Cancer Institute 93 (2001): 1088-1095.

Citation: Virgili E, Emili R, Hammarberg Ferri I, Palermo F, Calza L, Gardarelli M, Spina M. Lipidomics and Nutrylipidomics in Oncology: Review of the Literature. Journal of Bioinformatics and Systems Biology 1 (2018): 011014.

This article is an open access article distributed under the terms and conditions of the

Creative Commons Attribution (CC-BY) license 4.0 